\title{
Oral dexamethasone improved respiratory distress in children $<2$ years of age with acute bronchiolitis
}

Schuh S, Coates AL, Binnie R, et al. Efficacy of oral dexamethasone in outpatients with acute bronchiolitis.J Pediatr 2002 Jan;140:27-32.

\section{QUESTION: In children $<2$ years of age who present to the emergency department (ED) with acute bronchiolitis, what is the effectiveness of oral dexamethasone?}

\section{Design}

Randomised (allocation concealed*), blinded (clinicians, patients, data collectors, and outcome assessors),* placebo-controlled trial with follow up at 4 hours and 7 days.

\section{Setting}

The ED of a hospital in Toronto, Ontario, Canada.

\section{Patients}

71 children who were between 8 weeks and 23 months of age, had a first wheezing episode associated with respiratory distress and an upper respiratory tract infection, and had a Respiratory Disease Assessment Instrument rating $\geq 6$. Follow-up was $99 \%$ at 4 hours (mean age $6.5 \mathrm{mo}, 61 \%$ boys) and $94 \%$ at 7 days.

\section{Intervention}

36 children were allocated to a single dose of oral dexamethasone, $1 \mathrm{mg} / \mathrm{kg}$, and 34 were allocated to placebo. At 0, 30, 60, and 120 minutes, all children received albuterol (Ventolin $5 \%$ solution) via nebulizer, $2.5 \mathrm{mg}$ per dose $(0.5 \mathrm{ml})$ in $3 \mathrm{ml}$ normal saline and oxygen flow 6 to $7 \mathrm{l} / \mathrm{min}$. After a 4 hour observation period, children were discharged home and continued to receive either daily oral dexamethasone, $0.6 \mathrm{mg} / \mathrm{kg} /$ dose, or placebo for 5 days, in addition to nebulised albuterol, $1.5 \mathrm{mg}(0.3$ ml) 4 times per day.

\section{Main outcome measures}

Main outcome was Respiratory Assessment Change Score (RACS), which assesses changes in retractions, wheezing, and respiratory rate measured from 0 to 240 minutes (a decrease in RACS indicates improvement, and an increase indicates deterioration). Secondary outcomes included hospitalisation rates and RACS at 7 days.

\section{Main results}

Analysis was by intention to treat. Children who received dexamethasone had greater overall improvement in respiratory distress than did those who received placebo (mean RACS at $4 \mathrm{~h}-5.0 v-3.2, \mathrm{p}<0.03$ ). Fewer children who received dexamethasone had a poor response (ie, RACS $\leq-2$ ) than did those who received placebo. The rate of hospitalisation from the $\mathrm{ED}$ was lower in the dexamethasone group. The groups did not differ for mean RACS at 7 days $(-8.9 v-9.3, \mathrm{p}=0.75)$.

\section{Conclusion}

In children $<2$ years of age who presented to the emergency department with acute bronchiolitis, oral dexamethasone improved respiratory distress at 4 hours and reduced hospitalisations.

*See glossary.
Sources of funding: Medical Research Council of Canada and Merck Frosst Canada.

For correspondence: Dr S Schuh, The Hospital for Sick Children, Toronto, $O N$, Canada. Email suzanne.schuh@ sickkids.on.ca.
Oral dexamethasone vs placebo for acute bronchiolitis in children $<2$ years of aget

\begin{tabular}{lllll} 
Outcomes at $4 \mathrm{~h}$ & \multicolumn{2}{l}{ Dexamethasone Placebo } & RRR (95\% CI) & NNT (CI) \\
Poor response & $17 \%$ & $41 \%$ & $60 \%(11$ to 82$)$ & 5 (3 to 31$)$ \\
\hline Hospitalisation & $19 \%$ & $44 \%$ & $56 \%(9$ to 80$)$ & 5 (3 to 36$)$ \\
\hline
\end{tabular}

tAbbreviations defined in glossary; RRR, NNT, and $\mathrm{Cl}$ calculated from data in article.

\section{COMMENTARY}

Infants with acute bronchiolitis frequently present to pediatric EDs during the winter months and account for up to $17 \%$ of all infant hospitalisations in high prevalence areas. ${ }^{1}$ They have a variety of clinically indistinguishable pathologic conditions. Respiratory syncytial virus is associated with $60 \%$ of cases. ${ }^{2}$ Infants with first-time wheezing may be less likely to have asthma as an underlying cause than those with recurrent episodes. Most clinicians reserve glucocorticoids for recurring illness. Bronchodilators have yet to be definitively shown to be effective in decreasing hospitalisations from the ED in this population. ${ }^{3}$

Shuh et al restricted their methodologically strong study to infants with first-time wheezing of moderate severity presenting to an ED. They are the first to study glucocorticoid efficacy among non-hospitalised infants with presumed bronchiolitis. A family history of atopy, an indicator of asthmatic potential, was more prevalent in the dexamethasone group but did not affect the outcomes in a regression analysis.

Of the 2 dichotomous outcome measures, decreased hospitalisation rate is clearest in its clinical significance. The decreased hospitalisation achieved in the dexamethasone group was stable; only 1 delayed admission occurred among those discharged. Unscheduled cointerventions were more common in the placebo group. Adverse effects, although incompletely addressed, were not reported, despite the fact that the initial dose of dexamethasone was $40 \%$ higher than that used for croup. Although further study is warranted, many emergency clinicians and their patients are likely to accept an NNT as high as 36 to avoid hospitalisation to justify administering at least a single oral dose of dexamethasone to infants with first-time wheezing.

Peter C Wyer, MD New York Presbyterian Hospital New York City, New York

1 McConnochie KM, Roghmann KJ, Liptak GS. Hospitalization for lower respiratory tract illness in infants: variation in rates among counties in New York State and areas within Monroe County. J Pediatr. 1995;126:220-9.

Wright RB Pomerantz WI Luria JW New approathes to respiratory infections in children. Bronchiolitis and croup. Emerg Med Clin North Am 2002.20.93-114

Bronchiolitis and croup. Emerg Med Clin North Am 2002;20:93-114. Cochrane Database Syst Rev 2000;(2):CD001266. 